\title{
Oxidación de 2,5-dimetilfurano en un reactor de flujo
}

\author{
Katiuska Alexandrino, Ángela Millera, Rafael Bilbao, María U. Alzueta \\ Grupo de Procesos Termoquímicos (GPT) \\ Instituto de Investigación en Ingeniería de Aragón (I3A). \\ Universidad de Zaragoza, Mariano Esquillor s/n, 50018, Zaragoza, Spain. \\ Tel.+34-976762707, Fax+34-976762043, e-mail: katyalex@unizar.es
}

\section{Resumen}

El presente trabajo se centra en el estudio experimental y de modelado cinético de la oxidación a presión atmosférica de 2,5dimetilfurano. Los experimentos se llevan a cabo en un reactor de cuarzo de flujo pistón, bajo diferentes condiciones de operación, variando principalmente la temperatura, la concentración de oxígeno (estequiómetria) y de 2,5-dimetilfurano.

\section{Introducción}

Una de las técnicas utilizadas para reducir las emisiones provenientes del sector de transporte es la reformulación del combustible. En este campo, los compuestos oxígenados han sido propuestos para sustituir o ser usados como aditivo a los combustibles convencionales.

Algunos compuestos oxígenados propuestos ya han sido estudiados de forma individual y/o como aditivos en motores a gasolina y diésel [Yalavartthi y cols., 2013; Magnusson y Nilsson, 2011]. Sin embargo, para conocer el comportamiento que tienen estos compuestos durante la combustión, se hace necesario realizar experimentos de oxidación bajo condiciones de laboratorio muy bien controladas. Del mismo modo, el desarrollo de un modelo cinético-químico detallado es necesario para conocer los mecanismos de reacción por los cuales se produce la oxidación del compuesto oxigenado considerado.

El 2,5-dimetilfurano (2,5-DMF, $\mathrm{C}_{6} \mathrm{H}_{8} \mathrm{O}$ ), que se puede obtener por procesos catalíticos donde la materia prima es la fructosa, es uno de los compuestos oxígenados propuestos. Algunas de las propiedades que lo hacen atractivo para ser usado como biocombustible o aditivo son su alto punto de ebullición, su insolubilidad en agua y su alta densidad energética. De este modo, el presente trabajo se centra en el estudio de la oxidación de 2,5-DMF a presión atmosférica variando la temperatura, la estequiometría y la concentración de 2,5-DMF.

\section{Experimental}

Los experimentos se llevan a cabo en una instalación en fase gas a presión atmosférica, que consta de tres secciones: alimentación de gases, reacción, y de acondicionamiento y análisis de gases. Se utiliza un flujo total de gas de 1000 $\mathrm{mLN} / \mathrm{min}$, usándose $\mathrm{N}_{2}$ para obtener el caudal total, lo que resulta en un tiempo de residencia función de la temperatura de operación, $\mathrm{t}_{\mathrm{r}}(\mathrm{s})=195 / \mathrm{T}(\mathrm{K})$. Para la detección y cuantificación de los productos de oxidación analizados $\left(\mathrm{CO}, \mathrm{CO}_{2}\right.$ y $\left.\mathrm{H}_{2}\right)$ se usa un micro-cromatógrafo de gases (Agilent 3000A) y un analizador en continuo de $\mathrm{CO} / \mathrm{CO}_{2}$ (Uras 14 IR de ABB).

Los experimentos se realizan en el intervalo de temperatura de 800-1400 K, utilizando 100 y 300 ppm de 2,5-DMF. El parámetro empleado para describir la estequiometría es el índice de exceso de aire $(\lambda)$, el cual está definido por la ecuación E.1, y se varia su valor desde condiciones de pirólisis hasta muy oxidantes. La Tabla 1 sumariza las condiones experimentales utilizadas.

$\lambda=\frac{\left(\text { Aire }_{\text {Combustible }}\right)_{\text {real }}}{\left(\text { Aire }_{\text {Combustible }} \text { esta. }_{\text {atr }}\right.}=\frac{\text { aire }_{\text {real }}}{\text { air }_{\text {esta. }}}=\frac{O_{2, \text { real }}}{O_{2, \text { sta. }}}$

Donde:

$\lambda=0$ : Condiciones de pirólisis.

$\lambda<1$ : Condiciones reductoras.

$\lambda=1$ : Condiciones estequiómetricas.

$\lambda>1$ : Condiciones oxidantes.

\section{Modelado}

Las simulaciones se realizan con el programa informático CHEMKIN-II [Kee y cols. 1991], en conjunto con el código SENKIN [Lutz y cols., 1990]. El modelo cinético-químico detallado completo incluye 255 especies y 1314 reacciones.

\section{Resultados y discusión}

Los resultados indican que la temperatura influye sobre la oxidación de 2,5-DMF, siendo mayor el 
consumo de 2,5-DMF cuanto mayor es la temperatura.

El estudio de la influencia de la concentración de oxígeno indica que para $0 \leq \lambda \leq 1$ el perfil de concentración de 2,5-DMF no se modifica. Sin embargo, para $\lambda \geq 5$ el perfil de concentración de 2,5DMF se desplaza a temperaturas más bajas, siendo mayor el desplazamiento cuanto mayor es el valor de $\lambda$.

Por otro lado, los resultados para diferentes concentraciones de 2,5-DMF, y para $\lambda=0.3,1$ y 30 , indican que la concentración de 2,5-DMF ejerce influencia sobre su perfil de concentración únicamente para $\lambda=30$ ya que en este caso, el perfil de concentración se desplaza a temperaturas más bajas al usar una concentración superior de 2,5DMF. Es importante resaltar también que para la concentración de 2,5-DMF más elevada estudiada (300 ppm), y para $\lambda=0.3$ y 1 , además de $\mathrm{CO}, \mathrm{CO}_{2} \mathrm{y}$ $\mathrm{H}_{2}$, se forman otros productos como son el etileno, etano y acetileno, siendo mayores sus concentraciones cuanto menor es el valor de $\lambda$, es decir, cuanto menor es la concentración de oxígeno.

En general, el modelo cinético-químico desarrollado se ajusta bien a los datos experimentales. El análisis de velocidad de reacción realizado indica que el radical 5-metil-2-furanilmetilo $\quad\left(\mathrm{R}_{1} \mathrm{C}_{6} \mathrm{H}_{7} \mathrm{O}\right)$, originado por la abstracción de un hidrógeno del grupo metilo, es el más importante en el consumo de 2,5-DMF.

\section{Conclusiones}

El estudio de la oxidación de 2,5-DMF que se ha llevado a cabo en un reactor de flujo y bajo diferentes condiciones de operación, permiten concluir que tanto la temperatura, así como también la concentración de oxígeno y de 2,5-DMF influyen sobre la conversión de este compuesto. Se observa que una mayor temperatura favorece la oxidación de 2,5-DMF. Además, condiciones oxidantes y muy oxidantes desplazan el inicio de consumo de 2,5DMF a temperaturas más bajas. Por otro lado, bajo condiciones muy oxidantes, el aumento de la concentración de 2,5-DMF desplaza su perfil de concentración a temperaturas más bajas.

\section{Agradecimientos}

Los autores experesan su gratitud al Gobierno de Aragón, a MINECO y a FEDER (CTQ2012-34423) por la financiación concedida. K. Alexandrino agradece a MINECO por su beca pre-doctoral (BES-2013-063049).

\section{Referencias}

[1]. YALAVARTTHI, S., CHINTALAPUDI, A.K. and DEV, S. Performance and emissions analysis of diesel engine using oxygenated Compounds. International Journal of Advanced Science and Technology. 2013, 61, 9-16.

[2]. MAGNUSSON, R. and NILSSON, C. The influence of oxygenated fuels on emissions of aldehydes and ketones from a two-stroke apark ignition engine. Fuel. 2011, 90(3), 1145-1154.

[3]. KEE, R.J., RUPLEY, F.M. and MILLER, J.A. Chemkin-II: A Fortran chemical kinetics package for the analysis of gas-phase chemical kinetics. Livermore: Sandia National Laboratories, 1989. Report SAND89-8009B.

[4]. LUTZ, A., KEE R.J. and MILLER J.A. A Fortran program for predicting homogeneous gas phase chemical kinetics with sensitivity analysis. Livermore: Sandia National Laboratories, 1988. Report SAND878248.

\begin{tabular}{cccc}
\hline Exp. & $\lambda$ & $\begin{array}{c}2,5-\mathrm{DMF} \\
(\mathrm{ppm})\end{array}$ & $\begin{array}{c}\mathrm{O}_{2} \\
(\mathrm{ppm})\end{array}$ \\
\hline 1 & 0 & 100 & 0 \\
2 & 0.3 & 100 & 225 \\
3 & 0.7 & 100 & 525 \\
4 & 1 & 100 & 750 \\
5 & 5 & 100 & 3750 \\
6 & 30 & 100 & 22500 \\
7 & 0.3 & 300 & 675 \\
8 & 1 & 300 & 2250 \\
9 & 30 & 300 & 67500 \\
\hline
\end{tabular}

Tabla 1. Condiciones experimentales. $T=800-1400 \mathrm{~K} . \mathrm{Q}_{\mathrm{T}}=1000$ $\mathrm{mLN} / \mathrm{min} . \mathrm{t}_{\mathrm{r}}(\mathrm{s})=195 / \mathrm{T}(\mathrm{K}) . \mathrm{N}_{2}$ es usado para obtener el caudal total. 